OPEN ACCESS

Edited by:

Ye Shen,

University of Georgia, United States

Reviewed by:

Yue Liu,

Xiyuan Hospital, China

Zhe-Sheng Chen,

St. John's University, United States

*Correspondence:

Nian Li

linian@wchscu.cn

Specialty section:

This article was submitted to

Drugs Outcomes Research and

Policies,

a section of the journal

Frontiers in Pharmacology

Received: 17 June 2021

Accepted: 27 August 2021

Published: 16 September 2021

Citation:

Chen L, Yang $Y$, Fan J, Zhang $Y$ and Li N (2021) Trends of High-Impact Studies in Pharmacology and Pharmacy: A Cross-Sectional Study.

Front. Pharmacol. 12:726668

doi: $10.3389 /$ fphar.2021.726668

\section{Trends of High-Impact Studies in Pharmacology and Pharmacy: A Cross-Sectional Study}

\author{
Lingmin Chen ${ }^{1}$, Yi Yang ${ }^{2}$, Jin Fan ${ }^{3}$, Yonggang Zhang ${ }^{4,5}$ and Nian $\mathrm{Li}^{6 *}$ \\ ${ }^{1}$ Department of Anesthesiology and National Clinical Research Center for Geriatrics, West China Hospital, Sichuan University and \\ The Research Units of West China (2018RU012), Chinese Academy of Medical Sciences, Chengdu, China, ${ }^{2}$ Department of \\ Clinical Medicine, Gansu University of Traditional Chinese Medicine, Lanzhou, China, ${ }^{3}$ School of Health Preservation and \\ Rehabilitation, Chengdu University of Traditional Chinese Medicine, Lanzhou, China, ${ }^{4}$ Chinese Evidence-based Medicine Center, \\ West China Hospital, Sichuan University, Chengdu, China, ${ }^{5}$ Department of Periodical Press and National Clinical Research Center \\ for Geriatrics, West China Hospital, Sichuan University, Chengdu, China, ${ }^{6}$ Department of Medical Administration, West China \\ Hospital, Sichuan University, Chengdu, China
}

Objective: To investigate the trends of high-impact studies in pharmacology and pharmacy research and to provide evidence for future research in the field of pharmacology and pharmacy.

Methods: A cross-sectional study was performed to understand the current status of high-impact studies (top 1\%) in pharmacology and pharmacy research via InCites tool based on Web of Science Core Collection. VOSViewer software was used to visualize the results. The outcomes included development trends, countries, subject areas, research institutes, collaborative networks, and subject terms.

Results: We found 4,273 high-impact (top 1\%) studies between 2011 and 2020 in the field of pharmacology and pharmacy. The number of studies increased from 366 in 2011 to 510 in 2020. These studies were mainly distributed in the following Web of Science subject categories: pharmacology and pharmacy $(n=4,188)$; neurosciences $(n=397)$; chemistry, multidisciplinary $(n=359)$; chemistry, medicinal $(n=314)$; microbiology $(n=301)$; biotechnology and applied microbiology $(n=280)$. These studies were cited in 646,855 studies from more than 100 Web of Science subject categories, and studies in pharmacology pharmacy accounted for the largest share of these citations. The top three countries that contributed the highest number of studies were the United States, United Kingdom, and China. The top three institutions that contributed the highest number of studies in the United States were the University of California System, the National Institutes of Health $(\mathrm{NIH})$, and Harvard University. The top research collaborative circle was from universities in the United States. The top international collaborative circle was from universities from the United States, United Kingdom, Australia, and China. The subject-term analysis indicated that cancer was still the top disease, NF- $\mathrm{kB}$ was the top signaling pathway, and drug-delivery and nanoparticles were the top methods.

Conclusion: The high-impact studies in pharmacology and pharmacy research have grown over time. The United States, the United Kingdom, and China are the top countries that contributed the high-impact studies. Cancer is still the greatest challenge in the field of disease treatment. It calls for more international collaboration in pharmacology and pharmacy research, which will help discover novel drugs.

Keywords: high-impact studies, pharmacology and pharmacy research, visual analysis, cancer, InCites, Web of Science 


\section{INTRODUCTION}

Pharmacology is the study of the sources, uses, and mechanisms of action of drugs (Reidenberg, 1991; Barnes et al., 2005). Pharmacy is the science or practice of preparing, formulating, and dispensing medicinal drugs (Allen, 2012; Shcherbakova and Desselle, 2020). In the Web of Science subject categories, "pharmacology and pharmacy" is a category that includes studies from both fields. With the rapid development of research methods, a substantial number of studies were published in this category (Krämer, 2008; Jones, 2014). However, the trends of studies in this category are still unclear.

Trends of a research field are usually reflected by highimpact studies (Hollenberg et al., 2020). Therefore, analyzing these high-impact studies could help us identify the research trends and foresee the potential developments in this research field (Chen et al., 2020; De Serrano, 2017; Zhu et al., 2020). High-impact studies are essential manifestations of first-class research findings (Becker et al., 2018). Hence, analysis of highimpact studies will provide an objective reflection of the hot research spots and the focus areas in such research (Oberbauer, 2020), which will serve as a valuable reference for the development of similar research and assessments of the academic impact of these studies (Glynn and Greenland, 2020; Janke et al., 2019). Because of the rapid growth of digital publishing, we can easily access studies in different research fields (Zhu et al., 2020). Previously, several studies were published in different fields, including nursing (Zhu et al., 2020), hand surgery (Eberlin et al., 2012), and other fields (Zhang et al., 2019; Zhang et al., 2020). A nursing study by Zhu et al. (2020) has analyzed the high-impact studies in nursing based on the InCites database and provided references for the nursing managers in the research field. The InCites database is a scientific research performance analysis tool based on more than 30 years of authoritative citation data of the Web of Science Core Collection (Bornmann and Leydesdorff, 2013; Dardas et al., 2019; Zhu et al., 2020), which is superior for analyzing high-impact studies than directly searching the Web of Science Core Collection (Zhu et al., 2020). Up to now, there is no comprehensive analysis of the high-impact studies in pharmacology and pharmacy.

This cross-sectional study analyzed the high-impact studies in pharmacology and pharmacy using the InCites tool based on the Web of Science Core Collection. The objectives include reporting high-impact studies' dynamic trends, analyzing subject areas of highimpact studies, identifying top countries and institutions, exploring collaborative networks, and discovering hot research topics.

\section{METHODS}

\section{Study Design}

A cross-sectional study was conducted.

\section{Inclusion and Exclusion Criteria}

The inclusion criteria were as follows: 1) the research category should be "pharmacology and pharmacy" when using the InCites tool based on the Web of Science Core Collection (Zhu et al., 2020); 2) the article type should be article or review; 3 ) studies should be in the top $1 \%$; 4 ) studies should be published in the past 10 years, from 2011 to 2020 . The exclusion criteria were as follows: 1) article type was editorial, correction, or other types; 2) studies could not be found in the Web of Science Core Collection.

\section{Study Collection and Data Extraction}

The initial retrieval date was April 19, 2021. We performed a search using InCites tool based on the Web of Science Core Collection. The following filters were used: research area $=$ "Pharmacology and Pharmacy," year = "2011-2020," document type $=$ "article or review," study rank = "top 1\%". A total of 4,273 high-impact (top 1\%) studies were included. The following data were downloaded from the InCites tool: author names, title, journal, organization, keywords, references, page numbers, Category Normalized Citation Impact (CNCI) (Zhu et al., 2020), and country. The 4,273 studies were then searched in the Web of Science Core Collection via the InCites tool. The details were extracted, including author names, title, journal, organization, keywords, references, page numbers, and subject categories.

\section{Statistical Analysis}

Statistical analysis was performed using the following software: Excel and VOS Viewer. The outcomes included trends in the number and citation impact, subject areas, countries, institutions, collaborative networks, and subject terms. The citation impact was assessed using CNCI (Zhu et al., 2020), an indicator of the Web of Science for evaluating impact. A $\mathrm{CNCI}$ value of one indicates a citation performance on par with the world average, whereas CNCI values above and below one are considered above average and below average, respectively. A descriptive analysis was performed to assess the trends in the number and citation impact, subject areas, countries, and institutions. A visualization analysis was performed using VOS Viewer to evaluate the collaborative networks and subject terms.

\section{RESULTS}

\section{Trends in the Number and Citation Impact of High-Impact Studies in Pharmacology and Pharmacy Research}

From 2011 to 2020, the number of high-impact pharmacology and pharmacy research studies increased from 495 to 613 . The CNCI values decreased from 18.26 in 2011 to 14.19 in 2019 and dramatically increased in 2020 to 36.71 (Figure 1).

\section{Subject Categories of High-Impact Studies in Pharmacology and Pharmacy Research}

The 4,273 high-impact studies were distributed to more than 100 Web of Science subject categories. Table 1 shows the $11 \mathrm{Web}$ of 


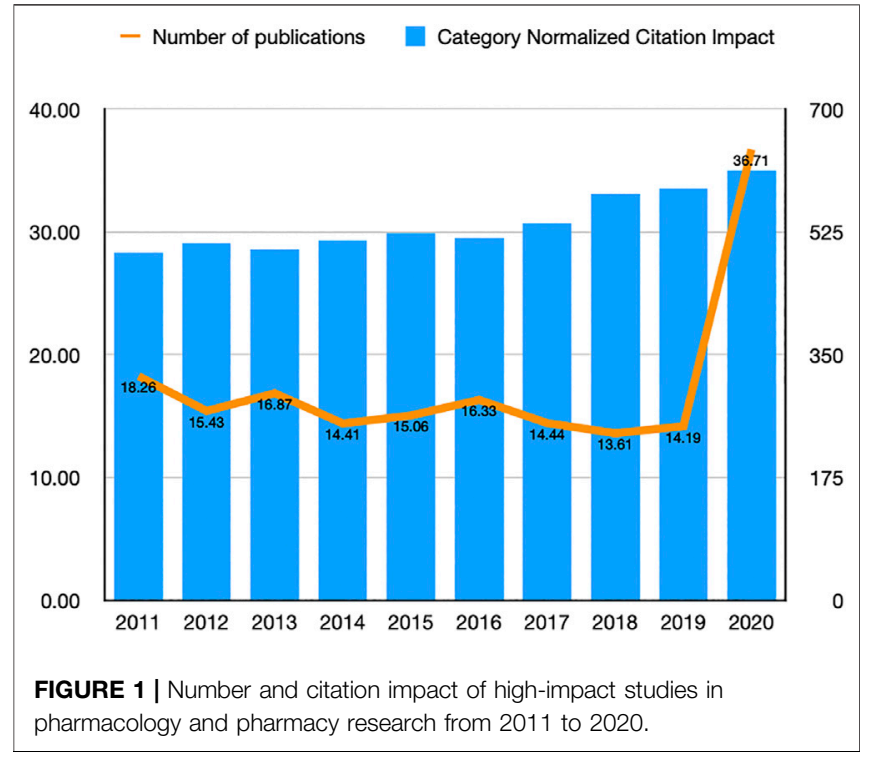

TABLE 1 | Eleven WOS subject categories that had more than 100 high-impact papers in pharmacology.

\begin{tabular}{llc} 
Ranking & \multicolumn{1}{c}{ Category } & Number of studies \\
\hline 1 & Pharmacology and pharmacy & 4,188 \\
2 & Neurosciences & 397 \\
3 & Chemistry, multidisciplinary & 359 \\
4 & Chemistry. medicinal & 314 \\
5 & Microbiology & 301 \\
6 & Biotechnology and applied microbiology & 280 \\
7 & Psychiatry & 261 \\
8 & Toxicology & 235 \\
9 & Infectious diseases & 166 \\
10 & Clinical neurology & 136 \\
11 & Medicine, research, and experimental & 113
\end{tabular}

Science subject categories with more than 100 studies each. After pharmacology and pharmacy $(n=4,188)$, neurosciences had the highest number of studies $(n=397)$, followed by chemistry, multidisciplinary $(n=359)$; chemistry, medicinal $(n=314)$; microbiology $(n=301) ;$ biotechnology and applied microbiology $(n=280)$. The 4,273 high-impact studies were cited by 646,855 studies, which were distributed in more than 100 Web of Science subject categories. The top 10 subject categories with the highest number of studies citing pharmacology and pharmacy research are shown in Table 2. Again, pharmacology and pharmacy accounted for the largest number of citations, with 124,423 citing studies. The citation impact of these high-impact studies extended to the categories of biochemistry and molecular biology $(n=72,550)$; chemistry, multidisciplinary $(n=53,574)$; neurosciences $(n=43,786)$; oncology $(n=36,180)$; chemistry, medicinal $(n=35,683)$; cell biology $(n=32,466)$; medicine, research, and experimental ( $n=30,207)$; multidisciplinary sciences $(n=28,415)$; nanoscience and nanotechnology $(n=27,474)$.
TABLE 2 | Top 10 WOS subject categories in terms of the number of studies citing the high-impact studies in pharmacology and pharmacy research.

\begin{tabular}{lll} 
Ranking & \multicolumn{1}{c}{ Category } & Number of studies \\
\hline 1 & Pharmacology and pharmacy & 124,423 \\
2 & Biochemistry and molecular biology & 72,550 \\
3 & Chemistry, multidisciplinary & 53,574 \\
4 & Neurosciences & 43,786 \\
5 & Oncology & 36,180 \\
6 & Chemistry, medicinal & 35,683 \\
7 & Cell biology & 32,466 \\
8 & Medicine, research, and experimental & 30,207 \\
9 & Multidisciplinary sciences & 28,415 \\
10 & Nanoscience nanotechnology & 27,474
\end{tabular}

\section{Countries of Origin of the High-Impact Studies in Pharmacology and Pharmacy Research}

The countries that published the most high-impact studies in pharmacology and pharmacy research were the United States, United Kingdom, China, Germany, and Italy. In terms of CNCI values, the top five countries were France, Australia, Sweden, Denmark, and United Kingdom (Table 4 and Figure 2).

\section{Institutions of High-Impact Studies in Pharmacology and Pharmacy Research}

The institutions that published the largest number of high-impact studies in pharmacology and pharmacy worldwide were the University of California System, National Institutes of Health (NIH), USA, Harvard University, University of London, and Institut National de la Sante et de la Recherche Medicale (INSERM). The total number of pharmacology and pharmacy research studies published by the top 20 originating institutions and their CNCI values is shown in Table $\mathbf{4}$ and Figure 3.

\section{Collaborative Networks of High-Impact Studies in Pharmacology and Pharmacy Research}

The collaborative networks were developed using VOS Viewer software. From the country level, four international collaborative circles were observed, among which the USA and China ranked first (red in Figure 4). Germany, France, and the Netherlands ranked second (green in Figure 4). Alexander Stephen ranked first at the author level, with 19 papers, 3,977 citations, and 178 links (Figure 5). The Journal of Controlled Release ranked first at the journal level, with 519 papers, 63,907 citations, and 964 links (Figure 6). At the institution level, there were four major collaborative circles (Figure 7). The largest node was the group of universities from the United States (red in the figure), including the University of Harvard, University of California System, University of Michigan, and John Hopkins University. The largest node of international collaborations was based on universities from England, Australia, the USA, and China. 


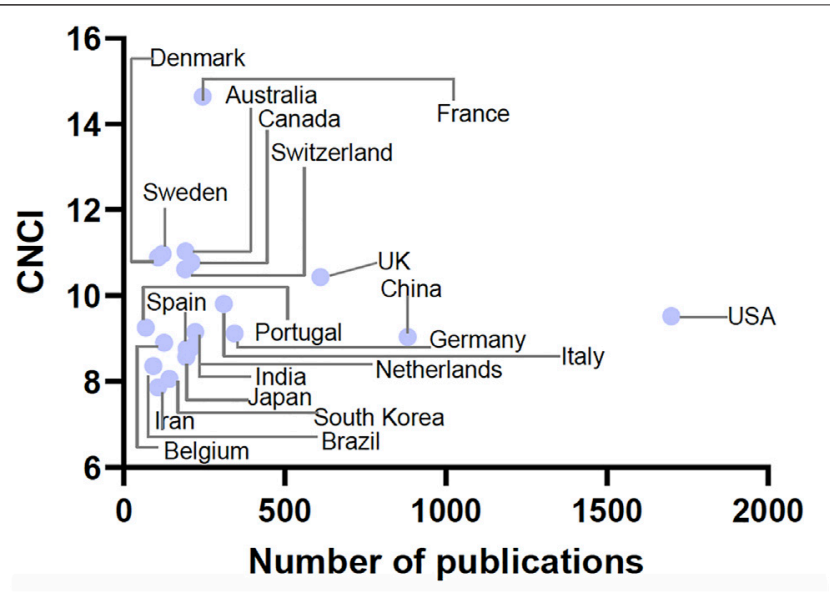

FIGURE 2 | Number of high-impact papers in pharmacology and pharmacy research and $\mathrm{CNCl}$ value by country.

\section{Heatmap of Subject Terms of High-Impact Studies in Pharmacology and Pharmacy Research}

In Figure 8, the heatmap of subject terms indicated that "in vitro," "expression”, "drug-delivery," "cells," "in vivo," "oxidative stress," "nanoparticles," "therapy," “activation," “apoptosis," “cancer," "inhibition," "NF-kappa-b," "mechanisms," "delivery resistance," "efficacy," "inflammation," and "identification" were the core themes that received the most attention from researchers in pharmacology and pharmacy.

\section{DISCUSSION}

Analyzing high-impact studies helps understand the trends in a research field (Zhu et al., 2020); however, no such study has been performed in the field of pharmacology and pharmacy. Thus, we performed the current cross-sectional study. In this study, we enrolled 4,273 high-impact (top 1\%) studies between 2011 and 2020 in pharmacology and pharmacy. The results indicated that the number of high-impact studies steadily increased from 2011 to 2020 . The number of studies published in 2020 was the highest, which was quite different from a previous study on nursing (Zhu et al., 2020). In that study, studies in the latest years were less than those in the earlier years. Because citations were cumulated year by year, studies published in the latest years usually have a lower impact and lesser citations. In our analysis, studies in 2020 accounted for the highest yearly number of studies. This might be caused by the COVID-19 pandemic. The scientific field has paid more attention to the treatment of diseases, while the pharmacology and pharmacy field plays a vital role in treating diseases (Rizk et al., 2020; Rawat et al., 2021; Ghasemnejad-Berenji and Pashapour, 2021). Thus, studies got more attention and citations. Based on CNCI values, the citation impact of high-impact studies in pharmacology and pharmacy research was much higher than the global average of the citation impact of studies. Therefore, it might be inferred that academic interest in high-quality research in pharmacology and pharmacy is increasing over time.

The objective measures of citation analysis can be used to assess the value of published research findings (Zhu et al., 2020). The interactions between research areas could be measured by analyzing the variations in the subjects and citing studies. In our study, pharmacology and pharmacy studies were distributed in more than 100 WOS subject categories, including pharmacology and pharmacy; neurosciences; chemistry, multidisciplinary; chemistry, medicinal; microbiology. The high-impact studies were cited by research from more than 100 WOS subject categories, which suggests a wide range of influence (Oelgemöller and Hoffmann, 2016). Among the top subject categories, microbiology ranked fifth, which might be because of the COVID-19 pandemic (Rawat et al., 2021). From the top citing subject categories, neurosciences and oncology ranked in the top 5, suggesting that diseases from such fields are important. Our results showed that the high-impact studies have an influence on a wide range of subject categories. This interdisciplinary impact could promote more academic research and ensure its sustained development.

In our study, the countries that contributed the highest number of high-impact studies were the USA, UK, China, Germany, and Italy. Moreover, the highest CNCI values of high-impact studies were France, Australia, Sweden, Denmark, and the United Kingdom. The values of the seven countries were greater than 10, which means that these countries contribute most to the increase in CNCI value in 2020. The difference in countries between the number of studies and CNCI values could reveal the scientific impact of different countries in this field (Diaz et al., 2021; Xie et al., 2021).

Analyzing the institutions allows identifying the research institutions with the greatest attractiveness of talents (Zhu et al., 2020). Our results suggested that the University of California System had the most high-impact studies $(n=233)$. Although Utrecht University, INSERM, and the University of Nottingham did not publish the most high-impact studies, their CNCI values ranked in the top 3, and all were more than 20. Therefore, these universities or institutes might attract talents in such fields (Diaz et al., 2021; Xie et al., 2021). Notably, the CNCI value was relatively low for the United States. However, in Table 4, more than $50 \%$ of institutes from the USA were the top 20 institutes with relatively high CNCI values. The possible reason might be that, as shown in Table 3, the number of publications in the USA was 1700, and the total number of citations was 297,545 . Table 4 shows that the number of USA publications of 11 institutions in the top 20 was 1,170 , and the total number of citations was 244,868 . The number of publications of the 11 institutions accounted for approximately 0.68 of the total USA publications, while the total number of citations accounted for 0.823 of the total number of USA citations. In other words, the number of studies published by other institutions from the USA accounted for only 0.32 , and the number of citations only accounted for 0.177 . This indicated that the number of citations of other institutions in the USA was relatively low. Therefore, the comprehensive calculation would lower the overall CNCI value, which would explain the relatively 


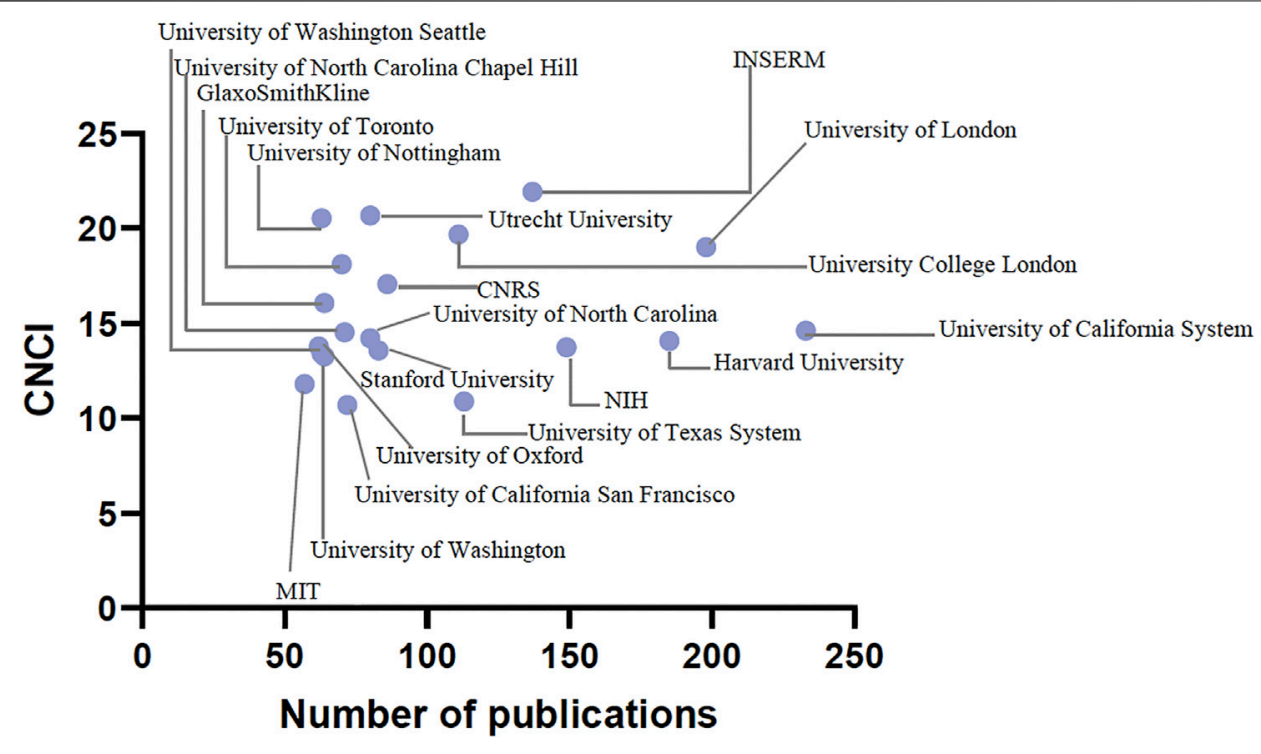

FIGURE 3 | Number of high-impact studies in pharmacology and pharmacy research and CNCl values of the top 20 institutions.

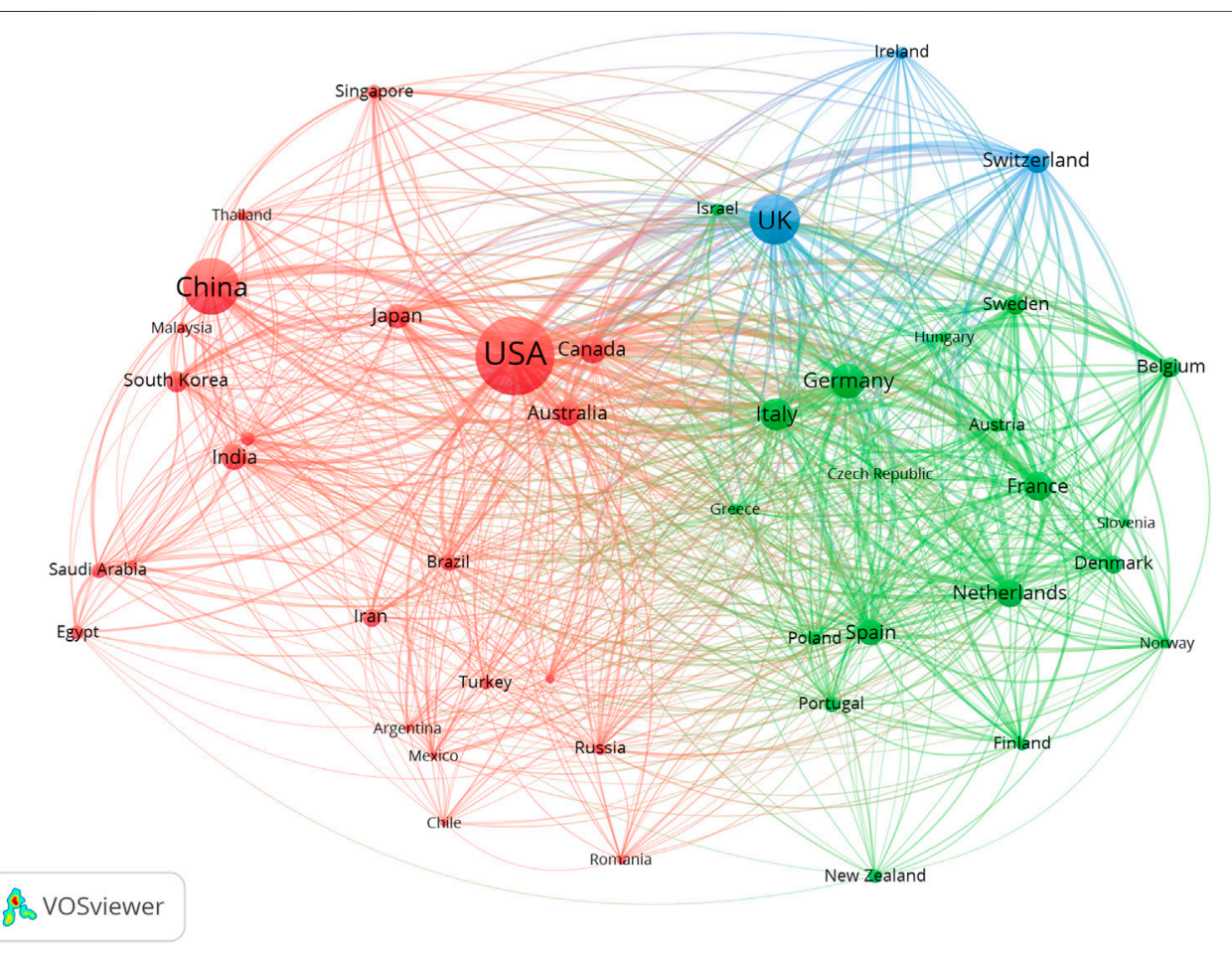

FIGURE 4 | Map of collaborative networks among a country of high-impact papers in pharmacology and pharmacy research around the world.

low CNCI values in Table 3 and the relatively high CNCI values in Table 4.

In the collaborative network analysis, the results suggested that the largest research collaborative node was the group of universities in the United States. Hence, these universities collectively possessed the most significant influence and research capacity, and were the core research institutions. In addition, the geographical distribution of these institutions indicated the same region or neighboring regions, which is consistent with previous studies in the nursing field (Zhu et al., 2020). The largest node of international collaborations was based on universities from England, Australia, the USA, and China, suggesting that international collaborations were critical in the top studies of such fields, which was consistent with the 


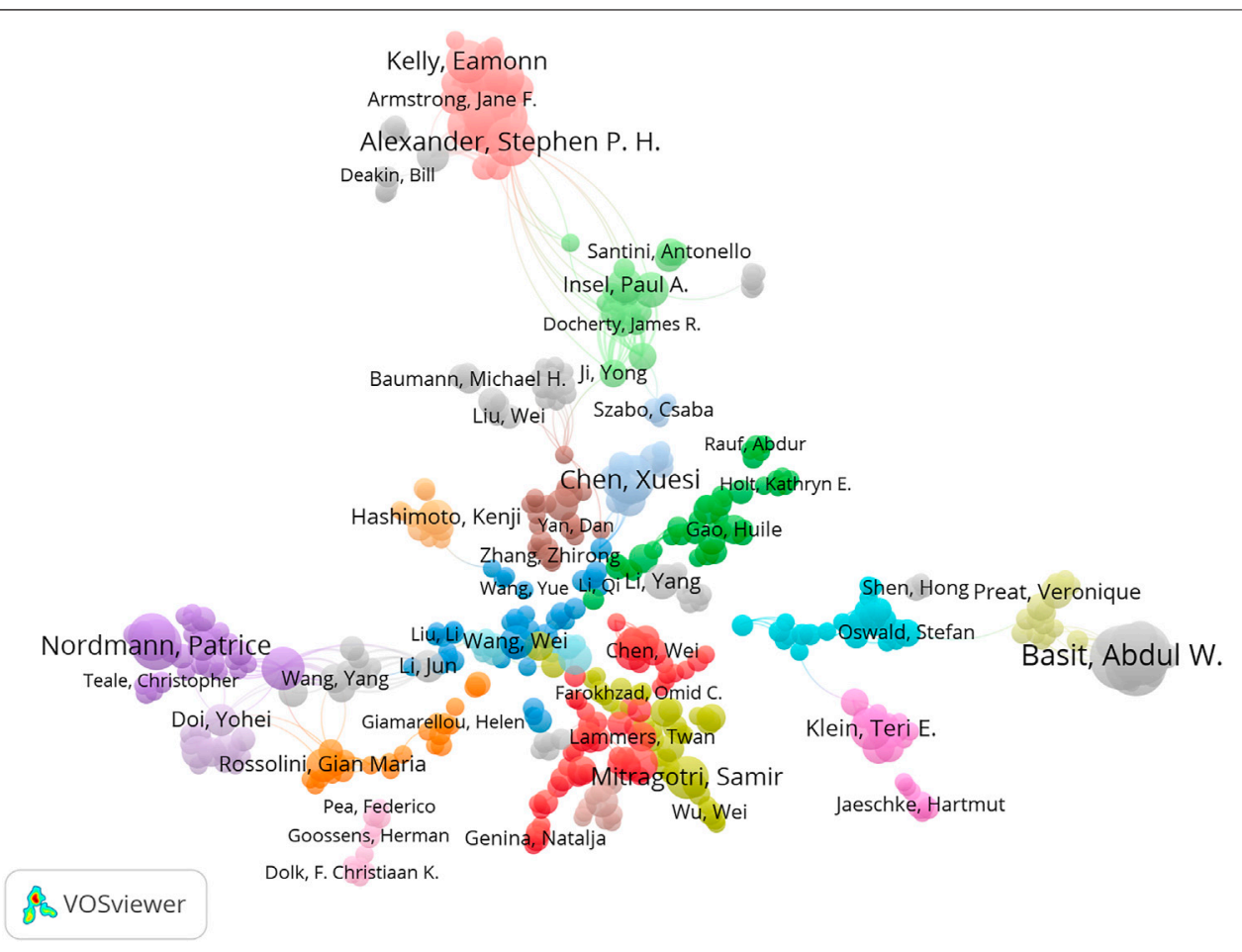

FIGURE 5 | Map of collaborative networks among the authors of high-impact papers in pharmacology and pharmacy research around the world.

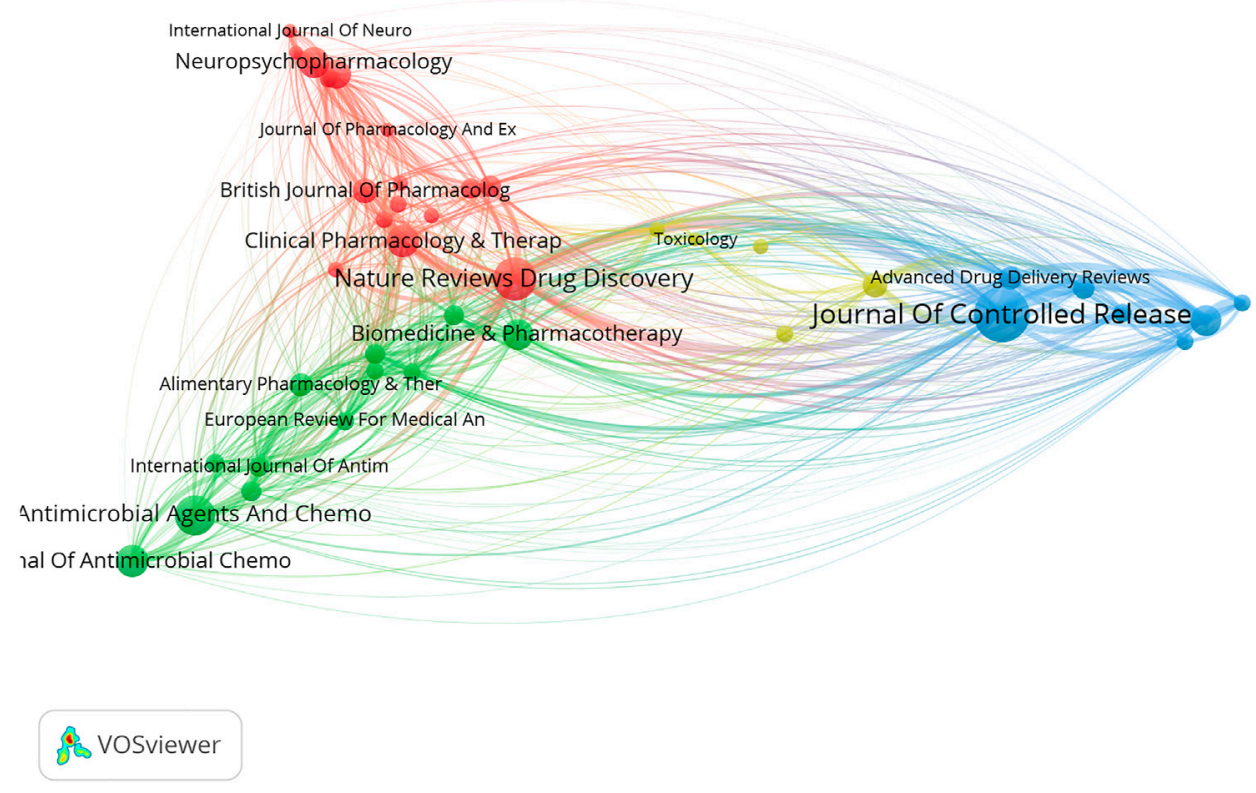

FIGURE 6 | Map of collaborative networks among journals of high-impact papers in pharmacology and pharmacy research around the world.

results of many studies (Chong et al., 2021; Li et al., 2021; Zhang S. et al., 2021). This indicates that the academic capacity of an institution is positively related to its cooperative relationship (Chong et al., 2021; Li et al., 2021; Zhang S. et al., 2021).
In the heatmap, we found that in vitro, expression, drug-delivery, cells, and in vivo ranked in the top five core themes in this field, suggesting that these were the essential concepts in this field. In addition, cancer had the most significant term frequency in the 


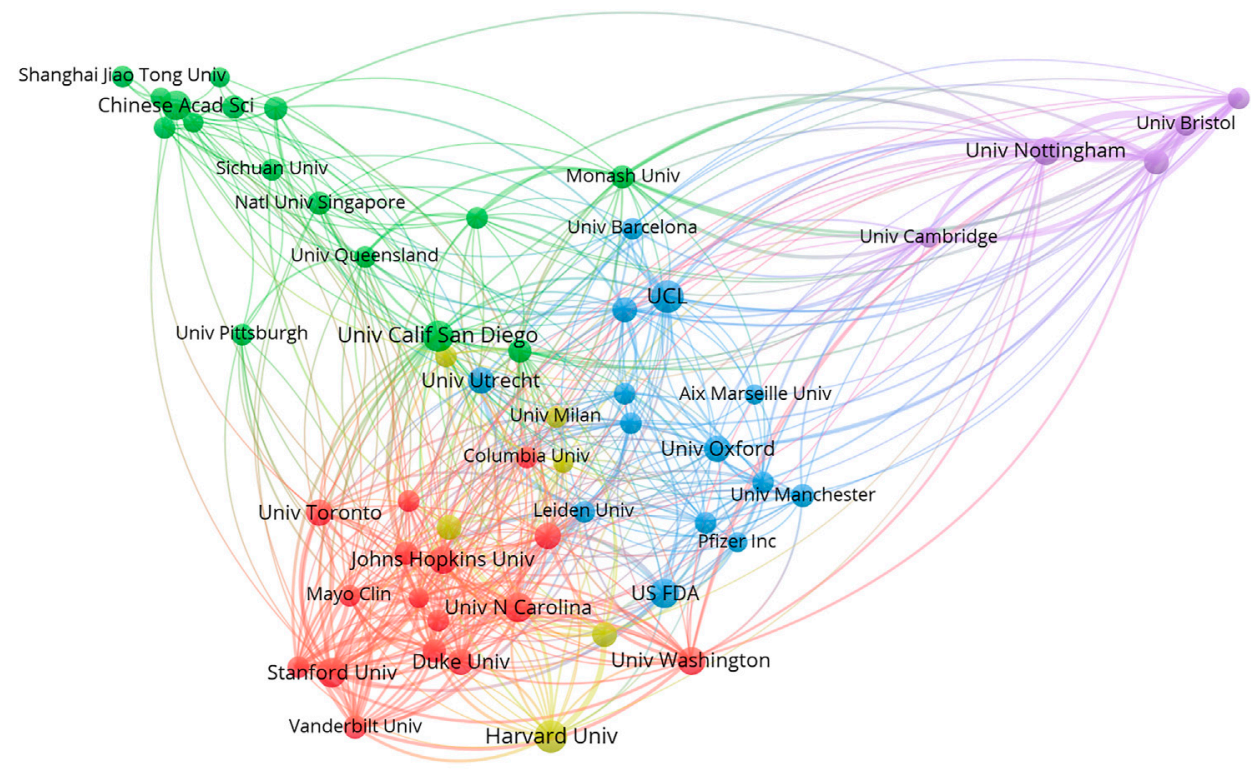

s vosviewer

FIGURE 7 | Map of collaborative networks among institutions of high-impact papers in pharmacology and pharmacy research around the world.

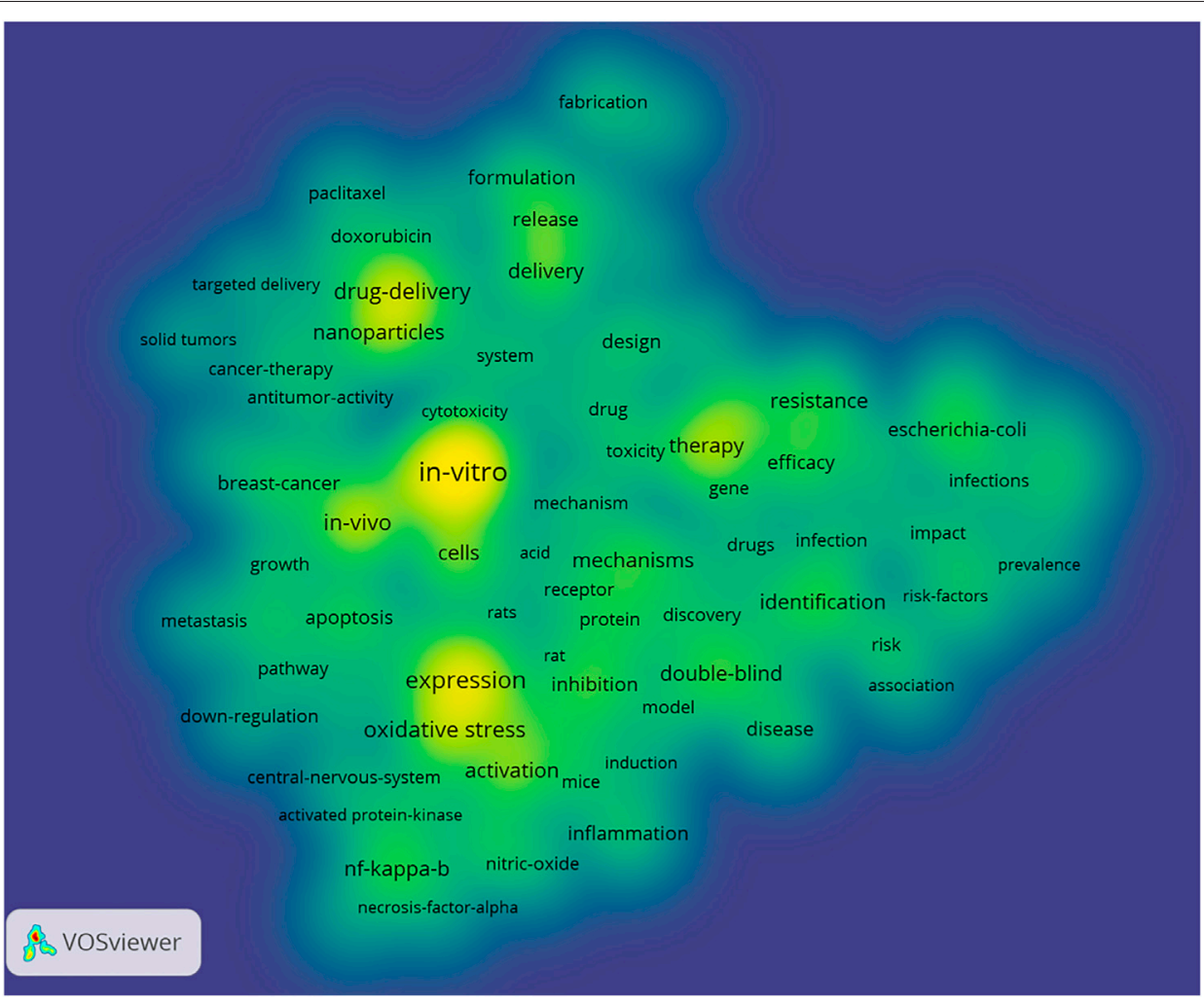

FIGURE 8 | Heatmap of subject terms of high-impact studies in pharmacology and pharmacy research. 
TABLE 3 | Number of high-impact papers in pharmacology and pharmacy and $\mathrm{CNCl}$ value by country.

\begin{tabular}{|c|c|c|c|c|}
\hline $\begin{array}{l}\text { Rank } \\
\text { of citation time }\end{array}$ & Country & Web of Science documents & Times cited & $\mathrm{CNCI}$ \\
\hline 1 & United States & 1,700 & 297,545 & 9.53 \\
\hline 2 & United Kingdom & 610 & 100,292 & 10.44 \\
\hline 3 & China & 882 & 75,638 & 9.05 \\
\hline 4 & Germany & 344 & 50,635 & 9.13 \\
\hline 5 & France & 245 & 41,317 & 14.64 \\
\hline 6 & Italy & 311 & 41,080 & 9.82 \\
\hline 7 & Canada & 211 & 39,815 & 10.78 \\
\hline 8 & Netherlands & 222 & 34,963 & 9.16 \\
\hline 9 & Switzerland & 190 & 34,012 & 10.62 \\
\hline 10 & Australia & 192 & 31,072 & 11.04 \\
\hline 11 & Japan & 194 & 29,757 & 8.58 \\
\hline 12 & India & 211 & 28,101 & 8.81 \\
\hline 13 & Spain & 195 & 25,146 & 8.77 \\
\hline 14 & Sweden & 121 & 23,138 & 10.98 \\
\hline 15 & Belgium & 125 & 22,160 & 8.91 \\
\hline 16 & Denmark & 106 & 20,855 & 10.90 \\
\hline 17 & South Korea & 142 & 16,643 & 8.07 \\
\hline 18 & Portugal & 68 & 10,827 & 9.26 \\
\hline 19 & Brazil & 92 & 10,750 & 8.37 \\
\hline 20 & Iran & 106 & 10,109 & 7.87 \\
\hline
\end{tabular}

TABLE 4 | Top 20 institutions of high-impact papers in pharmacology and pharmacy research.

\begin{tabular}{|c|c|c|c|c|}
\hline Rank & Institution & Web of Science documents & Times cited & $\mathrm{CNCl}$ \\
\hline 1 & University of California System & 233 & 39,918 & 14.62 \\
\hline 2 & $\mathrm{NIH}$ & 149 & 38,684 & 13.73 \\
\hline 3 & Harvard University & 185 & 37,733 & 14.07 \\
\hline 4 & University of London & 198 & 30,007 & 19.00 \\
\hline 5 & INSERM & 137 & 24,528 & 21.92 \\
\hline 6 & University of Texas System & 113 & 24,518 & 10.89 \\
\hline 7 & University of Washington & 64 & 16,249 & 13.27 \\
\hline 8 & University of Washington Seattle & 63 & 16,123 & 13.40 \\
\hline 9 & University of North Carolina & 80 & 15,612 & 14.21 \\
\hline 10 & MIT & 57 & 14,792 & 11.80 \\
\hline 11 & Utrecht University & 80 & 14,670 & 20.68 \\
\hline 12 & University of Toronto & 70 & 14,539 & 18.11 \\
\hline 13 & University College London & 111 & 14,066 & 19.68 \\
\hline 14 & University of North Carolina Chapel Hill & 71 & 13,926 & 14.54 \\
\hline 15 & University of Oxford & 62 & 13,855 & 13.78 \\
\hline 16 & Stanford University & 83 & 13,807 & 13.58 \\
\hline 17 & University of California San Francisco & 72 & 13,506 & 10.69 \\
\hline 18 & CNRS & 86 & 13,449 & 17.07 \\
\hline 19 & University of Nottingham & 63 & 13,355 & 20.53 \\
\hline 20 & GlaxoSmithKline & 64 & 12,289 & 16.07 \\
\hline
\end{tabular}

literature because it is still the greatest challenge in terms of treatment (Vasan et al., 2019). Cancer treatment is the most prominent topic for high-impact studies in this field (Jin et al., 2021; Li et al., 2020; Liu et al., 2020). We also found that oxidative stress and inflammation were the core themes, which predicted targets of drug therapy or drug discovery in the future (Reuter et al., 2010). Notably, the NF- $\kappa$ B pathway was among the core themes, suggesting that the signaling pathway is still a major pharmacology research topic (Eluard et al., 2020).

There were several limitations of this study. First, the InCites tool based on the Web of Science Core Collection was used to analyze the data. Data analysis from a second database might produce different results (Zhang S. et al., 2021). Second, only articles and reviews were included. The results might be biased because of enrolled types of studies, especially editorials from top scientists. Third, further analysis of the top 100 cited papers, authors, or institutions might provide meaningful results (Zhang et al., 2020; Zhang S. et al., 2021; Zhang Y. et al., 2021). Fourth, as there was no clear definition of high-impact papers, we included the top $1 \%$ studies. Other analytical criteria might be useful, such as analyzing the top $10 \%$ papers or the top 100 cited papers. Fifth, the data will change with time. Therefore, the conclusions should be updated in the future. 
Despite the limitations mentioned above, this study provides general trends in high-impact studies in pharmacology and pharmacy research published from 2011 to 2020. Our study suggests that high-impact studies have grown over time. The United States, United Kingdom, and China are the top countries that contributed the high-impact studies. Cancer is still the leading challenge in the field of disease treatment. It calls for more international collaboration in this field, which will help in the discovery of drugs.

\section{DATA AVAILABILITY STATEMENT}

The raw data supporting the conclusions of this article will be made available by the authors, without undue reservation.

\section{REFERENCES}

Allen, L. V., Jr. (2012). Remington: The Science and Practice of Pharmacy: from the Past into the Future. Int. J. Pharm. Compd. 16 (5), 358-362.

Barnes, J., Anderson, L. A., Gibbons, S., and Phillipson, J. D. (2005). Echinacea Species (Echinacea Angustifolia (DC.) Hell., Echinacea Pallida (Nutt.) Nutt.Echinacea Purpurea (L.) Moench): a Review of Their Chemistry, Pharmacology and Clinical Properties. J. Pharm. Pharmacol. 57 (8), 929-954. doi:10.1211/0022357056127

Becker, W. C., Bair, M. J., Picchioni, M., Starrels, J. L., and Frank, J. W. (2018). Pain Management for Primary Care Providers: A Narrative Review of HighImpact Studies, 2014-2016. Pain Med. 19 (1), 40-49. doi:10.1093/pm/ pnx146

Bornmann, L., and Leydesdorff, L. (2013). Macro-indicators of Citation Impacts of Six Prolific Countries: InCites Data and the Statistical Significance of Trends. PloS one 8 (2), e56768. doi:10.1371/ journal.pone. 0056768

Chen, Y., Yeung, A. W. K., Pow, E. H. N., and Tsoi, J. K. H. (2020). Current Status and Research Trends of Lithium Disilicate in Dentistry: A Bibliometric Analysis. The J. Prosthetic Dentistry. doi:10.1016/ j.prosdent.2020.08.012

Chong, Y., Long, X., and Ho, Y. S. (2021). Scientific Landscape and Trend Analysis of Keloid Research: a 30-year Bibliometric Review. Ann. Transl Med. 9 (11), 945. doi:10.21037/atm-21-508

Dardas, L. A., Woodward, A., Scott, J., Xu, H., and Sawair, F. A. (2019). Measuring the Social Impact of Nursing Research: An Insight into Altmetrics. J. Adv. Nurs. 75 (7), 1394-1405. doi:10.1111/jan.13921

De Serrano, L. O. (2017). Biotechnology of Siderophores in High-Impact Scientific fields. Biomol. Concepts 8 (3-4), 169-178. doi:10.1515/bmc-20170016

Diaz, A. P., Soares, J. C., Brambilla, P., Young, A. H., and Selvaraj, S. (2021). Journal Metrics in Psychiatry: What Do the Rankings Tell Us? J. Affect Disord. 287, 354-358. doi:10.1016/j.jad.2021.03.039

Eberlin, K. R., Labow, B. I., Upton, J., 3rd, and Taghinia, A. H. (2012). High-Impact Articles in Hand Surgery. Hand (New York, N,Y.) 7 (2), 157-162. doi:10.1007/ s11552-011-9388-7

Eluard, B., Thieblemont, C., and Baud, V. (2020). NF- $\kappa$ B in the New Era of Cancer Therapy. Trends Cancer 6 (8), 677-687. doi:10.1016/ j.trecan.2020.04.003

Ghasemnejad-Berenji, M., and Pashapour, S. (2021). Favipiravir and COVID-19: A Simplified Summary. Drug Res. (Stuttg) 71 (3), 166-170. doi:10.1055/a-12967935

Glynn, P., and Greenland, P. (2020). Contributions of the UK Biobank High Impact Papers in the Era of Precision Medicine. Eur. J. Epidemiol. 35 (1), 5-10. doi:10.1007/s10654-020-00606-7

Hollenberg, A. M., Bernstein, D. N., Baldwin, A. L., Beltejar, M. J., Rubery, P. T., and Mesfin, A. (2020). Trends and Characteristics of Spine Research from

\section{AUTHOR CONTRIBUTIONS}

YZ and NL designed the study and edited the article. YY searched the data. LC, YY, and JF analyzed the data. LC drafted the article. NL revised the article. All authors approved the final version of the article.

\section{FUNDING}

This study is partly supported by the Science and Technology Department of Sichuan Province (No: 2020YFS0186), China Postdoctoral Science Foundation (No: 2021M692274), The PostDoctor Research Project of West China Hospital of Sichuan University (No. 19HXBH071), National Natural Science Foundation of China (No: 82001130). This study is edited by Enliven.

2006 to 2015: A Review of Spine Articles in a High Impact General Orthopedic Journal. Spine (Phila Pa 1976) 45 (2), 141-147. doi:10.1097/ brs.0000000000003189

Janke, K. K., Kelley, K. A., Martin, B. A., Ray, M. E., and Sweet, B. V. (2019). Identifying High-Impact and Managing Low-Impact Assessment Practices. Am. J. Pharm. Educ. 83 (7), 7496. doi:10.5688/ajpe7496

Jin, H., Shi, Y., Lv, Y., Yuan, S., Ramirez, C. F. A., Lieftink, C., et al. (2021). EGFR Activation Limits the Response of Liver Cancer to Lenvatinib. Nature 595 (7869), 730-734. doi:10.1038/s41586-021-03741-7

Jones, D. S. (2014). The Journal of Pharmacy and Pharmacology 2013. J. Pharm. Pharmacol. 66 (1), 1. doi:10.1111/jphp.12210

Krämer, I. (2008). Pharmacy and Pharmacology of Biosimilars. J. Endocrinol. Invest. 31 (5), 479-488. doi:10.1007/bf03346394

Li, Q., Dai, W., Chen, X., Su, D., Yu, W., and Gu, X. (2021). Publication Trends and Hot Spots in Chronic Postsurgical Pain (CPSP) Research: A 10-Year Bibliometric Analysis. J. Pain Res. 14, 2239-2247. doi:10.2147/ JPR.S300744

Li, S., Liu, M., Do, M. H., Chou, C., Stamatiades, E. G., Nixon, B. G., et al. (2020). Cancer Immunotherapy via Targeted TGF- $\beta$ Signalling Blockade in TH Cells. Nature 587 (7832), 121-125. doi:10.1038/s41586-020-2850-3

Liu, X., Bao, X., Hu, M., Chang, H., Jiao, M., Cheng, J., et al. (2020). Inhibition of PCSK9 Potentiates Immune Checkpoint Therapy for Cancer. Nature 588 (7839), 693-698. doi:10.1038/s41586-020-2911-7

Oberbauer, R. (2020). Transplant Papers of High Impact Published in the Year 2019 in NDT. Nephrol. Dial. Transpl. 35 (4), 547-549. doi:10.1093/ndt/gfz233

Oelgemöller, M., and Hoffmann, N. (2016). Studies in Organic and Physical Photochemistry - an Interdisciplinary Approach. Org. Biomol. Chem. 14 (31), 7392-7442. doi:10.1039/c6ob00842a

Rawat, K., Kumari, P., and Saha, L. (2021). COVID-19 Vaccine: A Recent Update in Pipeline Vaccines, Their Design and Development Strategies. Eur. J. Pharmacol. 892, 173751. doi:10.1016/j.ejphar.2020.173751

Reidenberg, M. M. (1991). Clinical Pharmacology. JAMA 265 (23), 3107-3109. doi:10.1001/jama.1991.03460230057008

Reuter, S., Gupta, S. C., Chaturvedi, M. M., and Aggarwal, B. B. (2010). Oxidative Stress, Inflammation, and Cancer: How Are They Linked? Free Radic. Biol. Med. 49 (11), 1603-1616. doi:10.1016/j.freeradbiomed.2010.09.006

Rizk, J. G., Kalantar-Zadeh, K., Mehra, M. R., Lavie, C. J., Rizk, Y., and Forthal, D. N. (2020). Pharmaco-Immunomodulatory Therapy in COVID-19. Drugs 80 (13), 1267-1292. doi:10.1007/s40265-020-01367-z

Shcherbakova, N., and Desselle, S. (2020). Looking Back at US Pharmacy's Past to Help Discern its Future. Ann. Pharmacother. 54 (9), 907-920. doi:10.1177/ 1060028020911085

Vasan, N., Baselga, J., and Hyman, D. M. (2019). A View on Drug Resistance in Cancer. Nature 575 (7782), 299-309. doi:10.1038/s41586-019-1730-1

Xie, L., Lu, B., Ma, Y., Yin, J., Zhai, X., Chen, C., et al. (2021). The 100 Most-Cited Articles about the Role of Neurovascular Unit in Stroke 2001-2020: A Bibliometric Analysis. CNS Neurosci. Ther. 27 (7), 743-752. doi:10.1111/ cns. 13636 
Zhang, S., Fan, H., and Zhang, Y. (2021). The 100 Top-Cited Studies on Dyslexia Research: A Bibliometric Analysis. Front. Psychiatry 12, 714627. doi:10.3389/ fpsyt.2021.714627

Zhang, Y., Huang, L., Wang, Y., Lan, Y., and Zhang, Y. (2021). Characteristics of Publications on Occupational Stress: Contributions and Trends. Front. Public Health 9, 664013. doi:10.3389/fpubh.2021.664013

Zhang, Y., Quan, L., Xiao, B., and Du, L. (2019). The 100 Top-Cited Studies on Vaccine: a Bibliometric Analysis. Hum. Vaccin. Immunother. 15 (12), 3024-3031. doi:10.1080/21645515.2019.1614398

Zhang, Y., Xiong, Y., Cai, Y., Zheng, L., and Zhang, Y. (2020). The 100 Top-Cited Studies on Neuropsychology: A Bibliometric Analysis. Front. Psychol. 11, 550716. doi:10.3389/fpsyg.2020.550716

Zhu, R., Wang, Y., Wu, R., Meng, X., Han, S., and Duan, Z. (2020). Trends in HighImpact Papers in Nursing Research Published from 2008 to 2018: A Web of Science-Based Bibliometric Analysis. J. Nurs. Manag. 28 (5), 1041-1052. doi:10.1111/jonm.13038
Conflict of Interest: The authors declare that the research was conducted in the absence of any commercial or financial relationships that could be construed as a potential conflict of interest.

Publisher's Note: All claims expressed in this article are solely those of the authors and do not necessarily represent those of their affiliated organizations, or those of the publisher, the editors and the reviewers. Any product that may be evaluated in this article, or claim that may be made by its manufacturer, is not guaranteed or endorsed by the publisher.

Copyright (c) 2021 Chen, Yang, Fan, Zhang and Li. This is an open-access article distributed under the terms of the Creative Commons Attribution License (CC BY). The use, distribution or reproduction in other forums is permitted, provided the original author(s) and the copyright owner(s) are credited and that the original publication in this journal is cited, in accordance with accepted academic practice. No use, distribution or reproduction is permitted which does not comply with these terms. 